\title{
Calibration of CES functions for real-world multisectoral modeling
}

\author{
Ferran Sancho \\ Department of Economics \\ Universitat Autònoma de Barcelona \\ 08193-Bellaterra, Catalonia, Spain \\ ferran.sancho@uab.cat
}

\begin{abstract}
:
We show how to calibrate CES production and utility functions when indirect taxation affecting inputs and consumption is present. These calibrated functions can then be used in computable general equilibrium models. Taxation modifies the standard calibration procedures since any taxed good has two associated prices and a choice of reference value units has to be made. We also provide an example of computer code to solve the calibration of CES utilities under two alternate normalizations. To our knowledge, this paper fills a methodological gap in the CGE literature.
\end{abstract}

JEL classification: C63, C68, C81

Institutional support from research grants SEC200-712 and SGR2005-0712 is gratefully acknowledged. 


\section{Introduction}

Since its introduction by Arrow et al. (1961) the constant elasticity of substitution (CES) functions have become extremely popular in empirical research and in microeconomics textbooks. Functions with a constant elasticity of substitution are everywhere in economics for they provide a neat way to represent the technology and preference relations of economic agents. This is particularly so in computable general equilibrium (CGE) modeling where the CES formulation offers an unparalleled degree of flexibility in the modeling of substitution options among commodities and among inputs. From no substitution (the Leontief case of fixed coefficients) to perfect substitution (linearity) there is a whole range of possibilities for the CES functions to represent the curvature of convex isoquants and isoutilities.

CGE models are functional representations of the Walrasian canonical model where technology and preferences have a specific form that is suitable for numerical calculations as long as we assign numerical values to the parameters describing those functional forms. Consider the particular Cobb-Douglas case of a constants returns to scale production function with two inputs, labor $L$ and capital $K$, producing output $Y$. The calibrated function requires the knowledge of an efficiency parameter and two share coefficients, one for labor and one for capital. All in all, we need three parameters for full calibration. Available empirical data typically includes the value of output $p \cdot Y$ and the value of the two primary factors $w \cdot L$ and $r \cdot K$, that is, three observations. If we choose units in such a way that one unit of good is worth one unit of value, then $p=w=$ $r=1$ and $Y=L+K$. Notice then that the value of $Y$ is redundant. Notice too that because of constant returns to scale and cost minimization the share coefficient for labor determines that of capital. Summing up, two independent data observations are all we need to calibrate the two remaining independent parameters of the two inputs CobbDouglas production function. By duality the Cobb-Douglas cost function follows through. For the general Cobb-Douglas functions with $n$ inputs we only need $n$ independent data points. The general CES function with $n$ inputs, however, cannot be calibrated from the same data as the Cobb-Douglas function since it contains more parameters ( $n+1$ : 1 efficiency parameter, $n-1$ independent productivity coefficients and 1 elasticity value) than available independent observations ( $n$ ). We need an exogenous parameter and this is usually the elasticity of substitution which can be searched in the econometrics literature or else be directly estimated for the problem at hand. 
Another, and deeper, calibration problem has to do with the presence of indirect taxation in empirical databases. In an ideal textbook situation inputs will enter directly and without tax surcharges into the production or utility function. But in real world applications the value of inputs may be altered by the presence of indirect taxation. It is then necessary to modify the calibration techniques to clean up the data in such a way that only net of taxes inputs enter the production or utility function.

Calibration is a popular technique with CGE practitioners but because of its limited data requirements it is not so with econometricians (Mansur and Whalley, 1984). Its main appeal is that makes CGE models operational and tractable (Whalley, 1987) while at the same time guaranteeing consistency with the first order conditions of firms and consumers optimization problems. Despite its popularity and widespread use very few available references explain the intricacies of the calibration procedures. Rutherford (2002) and Nissen (2005) for static general equilibrium models and Klump and Saam (2006) for dynamic models are the exceptions to the rule. In all these cases, however, the explanations are in general restricted to textbook examples (2 goods, no taxes) that are of little use in practical empirical modeling based on actual databases (like inputoutput tables and social accounting matrices) with many goods and several indirect taxation categories.

Large scale multisectoral models are implemented using computing code. The code translates the economic theory underlying the model into a set of operational modules that are executable thanks to the numerical calibration. Calibration in turn is built upon the database and the available bank of elasticities depicting the economy. Calibration is globally successful when the constructed model is able to replicate the observed database as an equilibrium solution.

The technical details on calibration that we explain below are of course independent of the specific programming language used to implement a model. Some programming modules like GAMS-MPSGE (Rutherford, 1999) incorporate internally the calibration but in our opinion, debatable of course, knowing the details of the calibration is good theoretical training in the microeconomics of duality and this at the end gives the researcher more freedom to choose the programming tool (GAMS, MATLAB, MATHEMATICA, or even EXCEL), more control of the modeling options and more in- 
depth and hands-on knowledge of the data structure of the economy under consideration.

In Section II we discuss the calibration of CES technologies. Section III in turn is devoted to CES utilities. In both cases, and for notational simplicity, we start with simple no-tax cases and then use the developed structure to obtain the extensions to the more realistic cases with taxes. In Section IV we discuss how to deal with $n$ dimensional situations and provide some very common examples of calibrated functions in actual modeling. An Appendix presents GAMS code to illustrate the calibration of a CES function in the $n$ dimensional case.

\section{Calibration of CES production (or cost) functions}

For an $n$ input technology the CES production function is usually written as

$Y=\beta \cdot\left(\sum_{j=1}^{n} \alpha_{j} \cdot X_{j}^{\rho}\right)^{\frac{1}{\rho}}$

where $Y$ is an output, $X_{j}$ is an input, $\beta$ is an efficiency parameter, and $\alpha_{j}$ is a productivity parameter. The substitution parameter $\rho$ is related to the elasticity of substitution $\sigma$ by the relation $\sigma=1 /(1-\rho)$.

A somewhat more convenient way to write the CES function is

$Y=\left(\sum_{j=1}^{n}\left(\theta_{j} \cdot X_{j}\right)^{\rho}\right)^{\frac{1}{\rho}}$

thanks to the substitution

$\theta_{j}=\left(\beta \cdot \alpha_{j}\right)^{\frac{1}{\rho}}$

The full specification of (2) involves an assignment of values for the $n$ technical coefficients $\theta_{j}$ and the substitution parameter $\rho$. 
For the sake of expositional simplicity, let us consider a production function with two inputs and no indirect taxation of any kind affecting any of the inputs. From the first order conditions of the cost minimization problem

Min $\omega_{1} \cdot X_{1}+\omega_{2} \cdot X_{2} \quad$ subject to $Y=\left(\left(\theta_{1} \cdot X_{1}\right)^{\rho}+\left(\theta_{1} \cdot X_{1}\right)^{\rho}\right)^{\frac{1}{\rho}}$

where $\omega_{j}$ is the price of input $X_{j}$, it is possible to find the CES cost function

$C(Y)=\left(\left(\frac{\omega_{1}}{\theta_{1}}\right)^{\gamma}+\left(\frac{\omega_{2}}{\theta_{2}}\right)^{\gamma}\right)^{\frac{1}{\gamma}} \cdot Y$

In this expression we have $\gamma=\rho /(\rho-1)=1-\sigma$. Using Sheppard's Lemma we find conditional demand for inputs, say for input 1

$\frac{\partial C(Y)}{\partial \omega_{1}}=X_{1}=\frac{1}{\gamma} \cdot\left(\left(\frac{\omega_{1}}{\theta_{1}}\right)^{\gamma}+\left(\frac{\omega_{2}}{\theta_{2}}\right)^{\gamma}\right)^{\frac{1}{\gamma}-1} \cdot \gamma \cdot\left(\frac{\omega_{1}}{\theta_{1}}\right)^{\gamma-1} \cdot \frac{1}{\theta_{1}} \cdot Y$

Taking (4) into account we can simplify (5) to obtain

$X_{1}=C(Y) \cdot\left(\left(\frac{\omega_{1}}{\theta_{1}}\right)^{\gamma}+\left(\frac{\omega_{2}}{\theta_{2}}\right)^{\gamma}\right)^{-1} \cdot\left(\frac{\omega_{1}}{\theta_{1}}\right)^{\gamma-1} \cdot \frac{1}{\theta_{1}}$

Now since from (4) again we know that

$C(1)=\left(\left(\frac{\omega_{1}}{\theta_{1}}\right)^{\gamma}+\left(\frac{\omega_{2}}{\theta_{2}}\right)^{\gamma}\right)^{\frac{1}{\gamma}}$

From here

$C(1)^{-\gamma}=\left(\left(\frac{\omega_{1}}{\theta_{1}}\right)^{\gamma}+\left(\frac{\omega_{2}}{\theta_{2}}\right)^{\gamma}\right)^{-1}$

Substituting (8) into (6) and solving for $\theta$ we obtain 
$\theta_{1}^{\gamma}=\frac{C(Y)}{X_{1}} \cdot \omega_{1}^{\gamma-1} \cdot C(1)^{-\gamma}$

In the process of calibration it is customary to select units of measurement in such a way that one unit of good is worth one unit of value. In other words we implicitly use units such that $\omega_{1}=1$ and $C(Y)=Y$. It follows that $C(1)=1$ and from here (9) reduces to

$\theta_{1}=\left(\frac{Y}{X_{1}}\right)^{\frac{1}{\gamma}}$

In the numerator we have total output $Y$, alternatively, its value from the database, and the denominator is total input for 1 (or again, its value from the database). For the second input, a similar expression can be derived, and likewise for the $n$-input CES production function. In general

$\theta_{j}=\left(\frac{Y}{X_{j}}\right)^{\frac{1}{\gamma}}=\left(\frac{\sum_{i=1}^{n} X_{i}}{X_{j}}\right)^{\frac{1}{\gamma}}$

In (11) we take advantage of the fact that no tax affects the purchase of inputs and the selection of units that makes all prices unitary. Hence $Y=\sum_{i=1}^{n} X_{i}$.

A simple numerical example to illustrate the procedure follows. Suppose that we read from an input-output table or a social accounting matrix (SAM) database that $Y=100$, $X_{1}=60$ and $X_{2}=40$. Further suppose that we know the elasticity of substitution between inputs to be $\sigma=0.8$. In this case $\rho=-0.25$ and $\gamma=0.2$. Using (11) we obtain $\theta_{1}=12.8601$ and $\theta_{2}=97,6563$. Replace now these coefficients' values, the substitution parameter $\rho$ and the baseline level of inputs in (2) and the baseline output $Y$ should be exactly replicated by the calibrated CES production function. As an additional check, perform now the substitution of values in the cost function (4) for the parameter $\gamma$, the coefficients $\theta_{j}$ and unitary input prices to verify that for the calibrated cost function the condition $C(Y)=Y$ does indeed hold true.

\section{II.1 Calibration with taxes}


When inputs are taxed the relevant price from the viewpoint of firms' decisions is the after-tax price. Suppose an ad-valorem tax on input $j$ is levied at rate $s_{j}$. Then the price of input $j$ inclusive of tax is $\omega_{j} \cdot\left(1+s_{j}\right)$. Let us use the notation $\omega_{j}^{s}=\omega_{j} \cdot\left(1+s_{j}\right)$ to denote the after-tax price for input $j$. For the two input case, the cost minimization problem is now

Min $\omega_{1}^{s} \cdot X_{1}+\omega_{2}^{s} \cdot X_{2} \quad$ subject to $Y=\left(\left(\theta_{1} \cdot X_{1}\right)^{\rho}+\left(\theta_{1} \cdot X_{1}\right)^{\rho}\right)^{\frac{1}{\rho}}$

The problem has exactly the same appearance as the original problem except for the significance of inputs' prices now. Thus the minimization problem has the same formal solution as before but instead of (9) we now have

$\theta_{1}^{\gamma}=\frac{C(Y)}{X_{1}} \cdot\left(\omega_{1}^{s}\right)^{\gamma-1} \cdot C(1)^{-\gamma}$

It is convenient to slightly rewrite this as

$\theta_{1}^{\gamma}=\frac{C(Y)}{\omega_{1}^{s} \cdot X_{1}} \cdot\left(\omega_{1}^{s}\right)^{\gamma} \cdot C(1)^{-\gamma}=\frac{C(Y)}{\omega_{1}^{s} \cdot X_{1}} \cdot\left(\omega_{1} \cdot\left(1+s_{1}\right)\right)^{\gamma} \cdot C(1)^{-\gamma}$

In selecting units for calibration we need now to decide whether we equate units to pretax or after-tax prices since these are the two prices affecting inputs. From the viewpoint of inputs we want to isolate the net price as the baseline price prior to any tax surcharges. This makes comparison of input prices in different tax simulations or regimes far easier. In addition it allows us to distinguish which part in the total change is due to a direct change in a tax rate, for instance, from the tax-induced change in prices via general equilibrium effects. In practice all this amounts to setting $\omega_{1}=1$. On the other hand, from the viewpoint of the output its price should be inclusive of all tax surcharges since this is the last price in the chain. This entails fixing the price of one unit of output to be equal to one; in other words $C(1)=1$ again. Finally notice that the remaining fraction in (12) has total cost in the numerator (i.e. $C(Y)$ ) whereas the denominator picks up gross of tax cost for input 1 (i.e. $\omega_{1}^{s} \cdot X_{1}$ ). These two are magnitudes that can be directly read from the empirical database. Therefore the selection of units for inputs and output yields 
$\theta_{1}=\left(\frac{C(Y)}{\omega_{1}^{s} \cdot X_{1}}\right)^{\frac{1}{\gamma}} \cdot\left(1+s_{1}\right)$

This expression generalizes in a straightforward way to any other inputs in the CES function. Provided we know, or are able to calculate, tax rates (13) is all we need to calibrate a CES production function.

Let us illustrate again with a hypothetical example. Assume once more a substitution elasticity of $\sigma=0.8$ (i.e. $\gamma=0.2$ ). Take $Y=100, X_{1}=50, X_{2}=35$ and let taxes on inputs 1

and 2 be $T_{1}=10, T_{2}=5$. The calibration of a CES production function to these data requires first the determination of the tax rates: $s_{1}=10 / 50, s_{2}=5 / 35$. Next we select output units such that $C(Y)=Y$ and inputs units such that $\omega_{1}=\omega_{2}=1$. The last step involves the calculation of total after-tax input cost. In our example and using (13) we would obtain

$$
\begin{aligned}
& \theta_{1}=\left(\frac{100}{(1+10 / 50) \cdot 50}\right)^{\frac{1}{0.2}} \cdot(1+10 / 50)=15.4321 \\
& \theta_{2}=\left(\frac{100}{(1+5 / 35) \cdot 35}\right)^{\frac{1}{0.2}} \cdot(1+5 / 35)=111.6071
\end{aligned}
$$

The replication check works fine once again. When we plug inputs net of taxes $\left(X_{1}=50\right.$, $X_{2}=35$ ) into the CES production function (2) for the corresponding substitution parameter $\rho$ and the just calibrated coefficients $\theta_{j}$ we indeed obtain total observed output $Y=100$. Similarly for the calibrated cost function.

\section{Calibration of CES utility (or expenditure) functions}

We now turn to CES preferences as typically represented by utility functions. As before let $\sigma$ be the elasticity of substitution and $\rho=(\sigma-1) / \sigma$ be the substitution parameter. Suppose too, for now, that there are no indirect taxes affecting consumption. The two good CES utility function takes the form

$$
U=\left(\left(\theta_{1} \cdot X_{1}\right)^{\rho}+\left(\theta_{2} \cdot X_{2}\right)^{\rho}\right)^{\frac{1}{\rho}}
$$


where $X_{\mathrm{j}}$ stands for consumption of good $j$. From the budget constrained utility maximization problem (with income level $m$ )

$\operatorname{Max} U=\left(\left(\theta_{1} \cdot X_{1}\right)^{\rho}+\left(\theta_{2} \cdot X_{2}\right)^{\rho}\right)^{\frac{1}{\rho}}$ subject to $p_{1} \cdot X_{1}+p_{2} \cdot X_{2}=m$

we can, after quite a bit of tedious algebraic manipulation, derive the Marshallian demand functions for consumption goods 1 and 2

$$
X_{j}=\frac{m}{p_{j}} \cdot \frac{p_{j}^{1-\sigma} \cdot \theta_{j}^{\sigma-1}}{\sum_{i=1}^{2} p_{i}^{1-\sigma} \cdot \theta_{i}^{\sigma-1}}=\frac{m}{p_{j}} \cdot \frac{p_{j}^{1-\sigma} \cdot \theta_{j}^{\sigma-1}}{\Delta}
$$

where we simplify the expression by setting $\Delta=\sum_{i=1}^{2} p_{i}^{1-\sigma} \cdot \theta_{i}^{\sigma-1}$.

We now want to establish a procedure to numerically specify the utility coefficients $\theta_{j}$ in (14) given empirical observations in a database and an exogenous elasticity of substitution value.

We first notice that from (15) we can compute the ratio of consumption expenditures

$$
\frac{p_{1} \cdot X_{1}}{p_{2} \cdot X_{2}}=\frac{p_{1}^{1-\sigma} \cdot \theta_{1}^{\sigma-1}}{p_{2}^{1-\sigma} \cdot \theta_{2}^{\sigma-1}}
$$

The left-hand side is a ratio that can be easily calculated from the empirical database. Let us denote this observable consumption expenditure ratio as $c$. If the selection of units follows the traditional convention of assigning one unit of good to one unit of value, that is, we set prices to unitary values, i.e. $p_{\mathrm{j}}=1$, then (16) becomes

$$
c=\left(\frac{\theta_{1}}{\theta_{2}}\right)^{\sigma-1}
$$

The specification of the two coefficients $\theta_{j}$ cannot however be completed from the knowledge of $c$ and $\sigma$ alone. There is a degree of freedom that can be closed in several ways since adding a condition on the coefficients will not alter the utility function. It is just a normalization that we can select in a way that turns out to be convenient. 
One option is to choose $\theta_{j}$ in the non-negative unit simplex

$\theta_{1}+\theta_{2}=1$

From (17) and (18) we can uniquely determine the utility coefficients. In particular for $\theta_{1}$ we obtain

$\theta_{1}=c^{\frac{1}{\sigma-1}} \cdot\left(1+c^{\frac{1}{\sigma-1}}\right)^{-1}$

A second option to eliminate the degree of freedom rests in the crucial fact that the associated expenditure function is also a utility function. It is the so-called money metric utility (Varian, 1992). In other words we may scale the utilities in such a way that the minimum expenditure to attain one unit of utility is one unit of value. If $e(p, u)$ stands for the associated expenditure function, we are selecting utility units in such a way that $e(p, u)=u$.

If we now solve the expenditure minimization problem for the CES utility function we would find

$e(p, u)=u \cdot\left(\left(\frac{p_{1}}{\theta_{1}}\right)^{1-\sigma}+\left(\frac{p_{2}}{\theta_{2}}\right)^{1-\sigma}\right)^{\frac{1}{1-\sigma}}$

Recall the previous definition of $\Delta$ and use it in (20) to obtain

$e(p, u)=u \cdot \Delta^{\frac{1}{1-\sigma}}$

For the isoutility labeled with value $u=1$ we have

$e(p, 1)=\Delta^{\frac{1}{1-\sigma}}$

or alternatively

$\Delta=e(p, 1)^{1-\sigma}$ 
From the Marshallian demand functions in (15) and condition (23) we can express the expenditure shares for each good as

$$
\alpha_{j}=\frac{p_{j} \cdot X_{j}}{m}=\frac{p_{j}^{1-\sigma} \cdot \theta_{j}^{\sigma-1}}{\Delta}=\frac{p_{j}^{1-\sigma} \cdot \theta_{j}^{\sigma-1}}{e(p, 1)^{1-\sigma}}
$$

Remember that we are implicitly choosing utility units such that $e(p, u)=u$. Thus $e(p, 1)=1$. On the other hand the conventional selection of consumption units entails that all prices are unitary, i.e. $p_{j}=1$. Plugging in all these values into (24) and bearing in mind that expenditure shares necessarily add up to 1 we obtain

$$
\alpha_{1}+\alpha_{2}=\theta_{1}^{\sigma-1}+\theta_{2}^{\sigma-1}=1
$$

Combining this restriction with condition (17) we can solve for the utility coefficients under this second normalization and find the following values

$$
\begin{aligned}
& \theta_{1}=\left(\frac{c}{1+c}\right)^{\frac{1}{\sigma-1}} \\
& \theta_{2}=\left(\frac{1}{1+c}\right)^{\frac{1}{\sigma-1}}
\end{aligned}
$$

We can now proceed to do the data replication check with some hypothetical numerical examples. Suppose that $\sigma=4$ and observed consumption demands are $C_{1}=90$ and $C_{2}=10$ (implicitly we take prices of goods 1 and 2 to be unitary and thus income $m=100$ ). If we use the first normalization option in (19) we would obtain coefficients

$$
\begin{aligned}
& \theta_{1}=0.6753 \\
& \theta_{2}=0.3247
\end{aligned}
$$

Using these utility coefficients in the Marshallian CES demand functions in (15) we can verify that observed consumptions are duly replicated by the demand function. When we use the second normalization set up in (26) we would generate coefficients

$$
\begin{aligned}
& \theta_{1}=0.9655 \\
& \theta_{2}=0.4642
\end{aligned}
$$


Observed demands are again replicated when we substitute these coefficients in (15) but with the added bonus that total associated expenditure now also measures total utility. Hence this second normalization makes welfare computations in counterfactual policy scenarios and simulations extremely simple.

\section{III.1 Calibration with taxes}

It is time now to introduce indirect taxes in our problem. For final purchases agents may face a sales tax, or a consumption based value-added tax. In this circumstance final prices should include the tax surcharge. If $v_{j}$ is the ad-valorem tax rate on final purchases of good $\mathrm{j}$, then the relevant price for consumers' decisions is $p_{j} \cdot\left(1+v_{j}\right)$. The CES utility maximization problem would now be

$\operatorname{Max} U=\left(\left(\theta_{1} \cdot X_{1}\right)^{\rho}+\left(\theta_{2} \cdot X_{2}\right)^{\rho}\right)^{\frac{1}{\rho}}$ subject to $p_{1} \cdot\left(1+v_{1}\right) \cdot X_{1}+p_{2} \cdot\left(1+v_{2}\right) \cdot X_{2}=m$

with solution

$$
\begin{aligned}
& X_{j}=\frac{m}{\left(1+v_{j}\right) \cdot p_{j}} \cdot \frac{\left(1+v_{j}\right)^{1-\sigma} \cdot p_{j}^{1-\sigma} \cdot \theta_{j}^{\sigma-1}}{\sum_{i=1}^{2}\left(1+v_{i}\right)^{1-\sigma} \cdot p_{i}^{1-\sigma} \cdot \theta_{i}^{\sigma-1}}= \\
& =\frac{m}{\left(1+v_{j}\right) \cdot p_{j}} \cdot \frac{\left(1+v_{j}\right)^{1-\sigma} \cdot p_{j}^{1-\sigma} \cdot \theta_{j}^{\sigma-1}}{\Delta}
\end{aligned}
$$

and $\Delta$ now being $\Delta=\sum_{i=1}^{2}\left(1+v_{i}\right)^{1-\sigma} \cdot p_{i}^{1-\sigma} \cdot \theta_{i}^{\sigma-1}$

As in the no tax case, given an elasticity value $\sigma$ calibration requires to determine the utility coefficients $\theta_{j}$ in a way that replication of observed data is verified. Without explicitly repeating all of the steps outlined above, for option 1 we want to specify coefficients that add up to one and for option 2 coefficients that satisfy that utility is directly expressed in terms of expenditure.

For the first case, using (27) we see that the consumption expenditure ratio will now be

$$
c=\frac{\left(1+v_{1}\right) \cdot p_{1} \cdot X_{1}}{\left(1+v_{2}\right) \cdot p_{2} \cdot X_{2}}=\frac{\left(\left(1+v_{1}\right) \cdot p_{1}\right)^{1-\sigma} \cdot \theta_{1}^{\sigma-1}}{\left(\left(1+v_{2}\right) \cdot p_{2}\right)^{1-\sigma} \cdot \theta_{2}^{\sigma-1}}
$$


When selecting units such that prices are unitary and making

$\left(\frac{1+v_{1}}{1+v_{2}}\right)^{1-\sigma}=k$

we obtain

$c=k \cdot\left(\frac{\theta_{1}}{\theta_{2}}\right)^{\sigma-1}$

Condition (30) plus (18) and some algebra yields

$\theta_{1}=\left(\frac{c}{k}\right)^{\frac{1}{\sigma-1}} \cdot\left(1+\left(\frac{c}{k}\right)^{\frac{1}{\sigma-1}}\right)^{-1}$

Notice that in the no tax situation the coefficient $k=1$ and (31) reverts to (19).

For the second option, the normalization that equates expenditure and utility levels, we proceed as follows. The CES expenditure function would now take the form

$e(p, u)=u \cdot\left(\left(1+v_{1}\right)^{1-\sigma} \cdot\left(\frac{p_{1}}{\theta_{1}}\right)^{1-\sigma}+\left(1+v_{2}\right)^{1-\sigma} \cdot\left(\frac{p_{2}}{\theta_{2}}\right)^{1-\sigma}\right)^{\frac{1}{1-\sigma}}=u \cdot \Delta^{\frac{1}{1-\sigma}}$

Under the utility normalization $e(p, u)=u$ and taking $u=1$ the expenditure shares can be seen to be

$\alpha_{j}=\frac{\left(1+v_{j}\right) \cdot p_{j} \cdot X_{j}}{m}=\frac{\left(1+v_{j}\right)^{1-\sigma} \cdot p_{j}^{1-\sigma} \cdot \theta_{j}^{\sigma-1}}{e(p, 1)^{1-\sigma}}$

If units are chosen such that pre-tax prices are unitary (34) becomes

$$
\alpha_{j}=\left(1+v_{j}\right)^{1-\sigma} \cdot \theta_{j}^{\sigma-1}
$$

Consumption shares add up to one. For the two goods case we have

$\left(1+v_{1}\right)^{1-\sigma} \cdot \theta_{1}^{\sigma-1}+\left(1+v_{2}\right)^{1-\sigma} \cdot \theta_{2}^{\sigma-1}=1$ 
We have obtained a non-linear system for the utility coefficients $\theta_{j}$ comprised of condition (35) plus condition (30). Solving for $\theta_{j}$ we would find the calibrated coefficients to be

$$
\begin{aligned}
& \theta_{1}=\left(1+v_{1}\right) \cdot\left(\frac{c}{1+c}\right)^{\frac{1}{\sigma-1}} \\
& \theta_{2}=\left(1+v_{2}\right) \cdot\left(\frac{1}{1+c}\right)^{\frac{1}{\sigma-1}}
\end{aligned}
$$

Let us now consider some numerical examples. Suppose that consumptions are $X_{1}=80$, $X_{2}=8$ with consumption taxes being, respectively, $V_{1}=10$ and $V_{2}=2$ and the elasticity of substitution in consumption is $\sigma=4$. Using solution (31) the calibrated CES utility coefficients would be

$\theta_{1}=0.6518$

$\theta_{2}=0.3482$

Replacing these coefficients in the Marshallian demand function (27) for the appropriate ad-valorem tax rates and total income yields the observed values for demand.

If instead of the normalization solution (31) we use the solution in (36) the calibrated coefficients would turn out to be

$\theta_{1}=1.0862$

$\theta_{2}=0.5802$

Once again observed demands can be seen to be correctly replicated by the calibrated function and, in addition, total expenditure can be seen to be equal to total utility for this configuration, i.e., $e(p, u)=u$ with $u=100$.

\section{Extensions}

The procedures outlined above apply to the two goods case. The conceptual extensions to the $n$ good case are straightforward and will not be pursued here in much more detail. Whereas on the production side expression (13) generalizes without any difficulties to the $n$ input case, there is one technical difficulty in the consumption case that involves 
the solution of the nonlinear system of utility coefficients. In the $n$ good case, for example, conditions (18) and (30) can be seen to take the following form

$$
\begin{aligned}
& \sum_{j=1}^{n} \theta_{j}=1 \\
& c_{i j}=k_{i j} \cdot\left(\frac{\theta_{i}}{\theta_{j}}\right)^{\sigma-1}
\end{aligned}
$$

In (30') $c_{i j}$ stands for the consumption expenditure ratio between goods $i$ and $j$ whereas the constants $k_{i j}$ represent the tax ratios $\left(\left(1+v_{i}\right) /\left(1+v_{j}\right)\right)^{1-\sigma}$.

Since there are only $n-1$ independent expenditure ratios, in (18') and (30') we have altogether $n$ equations and $n$ utility coefficients to be determined. Similar considerations apply to the second normalization. Along with (30') the general $n$ good case would also require the following condition to be fulfilled

$$
\sum_{j=1}^{n}\left(1+v_{j}\right)^{1-\sigma} \cdot \theta_{j}^{\sigma-1}=1
$$

Both systems can be solved using computational techniques and to this effect code written in GAMS is presented in the Appendix. The system of equations, as constructed, is a square system that falls within the class of Constrained Nonlinear Systems (CNS) and can be quickly solved using GAMS-CNS. The example uses a 4 good utility function but the reader can easily modify the problem to deal with higher dimensions. Or reformulate to lower dimensions and check the numerical values provided in the utility examples above. After solving the nonlinear model some replication computations are added and performed to verify that numerical calibration has proceeded as expected from the theory. The two normalization options are included and the user can select which one to use or, if needed, include other and different normalizations.

A different type of extension a modeler may need to deal with has to do with the way taxes are levied on production or consumption inputs. We have focus on ad-valorem taxes but there are cases where taxes are instead unitary. Indirect energy taxes are an example. If tax rates are unitary the above procedures can be quickly adapted to solve 
the calibration problem. A further complication is when a given commodity is taxed twice in a composite way. In Europe, for instance, gasoline purchases by consumers are taxed by a specific unitary gasoline tax and on top of that there is a value-added tax. The same applies to the purchase of alcoholic beverage purchases. Several recurrent taxes can therefore be dealt with the procedures outline above, although the underlying algebra presentation inevitably becomes a bit more cluttered. This is the counterpart to the flexibility to deal with several alternate tax situations.

As for content-specific applications, there are several possibilities that originate from available multisectoral databases (input-output tables or social accounting matrices) and the econometrics literature on elasticities of substitution. On the production side CES technologies are typically depicted when using the Armington (1969) assumption to model substitution between domestic and imported goods. CES technologies are also used in modeling substitution between primary factors-types of labor and types of capital, or between value-added and a composite primary energy input which in turn can be a CES aggregate of different energy goods. In most practical cases taxation (tariffs, labor taxes, output taxes, and the like) affecting the use of inputs into production will be present and thus we need procedures for calibration that take actual taxation protocols into account.

The calibration of CES functions is also relevant for sensitivity analysis. When there is uncertainty, or simply, lack of knowledge on the values of key elasticities, a range of sensible literature values can be selected and comparative simulations run. The CES approach offers us a degree of modeling flexibility way beyond that of the commonly adopted Cobb-Douglas functions. Even when unitary substitution elasticities are called for, the possibility to rerun alternate simulations is invaluable from the perspective of sensitivity analysis. 


\section{Appendix: GAMS code for CES utility calibration}

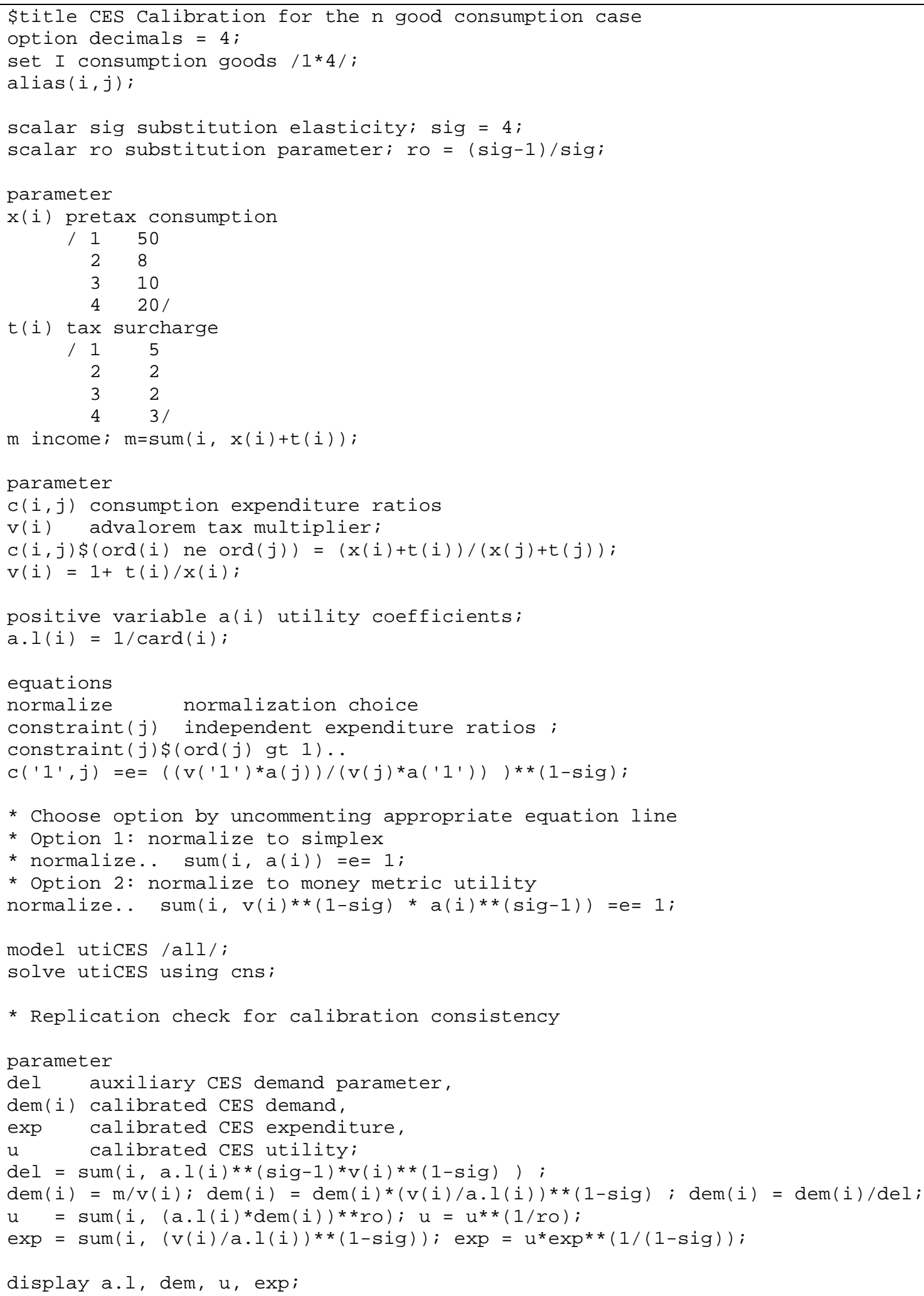




\section{References}

Armington, P.S. (1969), "A theory of demand for products distinguished by place of production”, International Monetary Fund Staff Papers, vol. 16, pp. 159-76.

Arrow, K.J, H.B. Chenery, B.S. Minhas and R.M. Solow (1961) "Capital-Labor Substitution and Economic Efficiency", The Review of Economics and Statistics, Vol. 43, No. 3, pp. 225-250.

Klump, R. and M. Saam (2006). "Calibration of Normalized CES Production Function in Dynamic Models”, Center for European Economic Research, Discussion paper 06078.

Mansur A. and J. Whalley (1984). "Numerical Specification of Applied General Equilibrium Models: Estimation, Calibration, and Data" in Herb Scarf and John Shoven (eds.), Applied General Equilibrium Analysis, Chapter 3 (Cambridge, Cambridge University Press).

Nissen, D. (2005). “A Note in Calibration of Cost and expenditure Functions”, mimeo, School for International and Public Affairs, Columbia University.

Rutherford, T. (1999). "Applied General Equilibrium Modeling with MPSGE as a GAMS Subsystem: An Overview of the Modeling Framework and Syntax," Computational Economics, 14, pp. 1-46.

Rutherford, T. (2002). "Lecture Notes on Constant Elasticity Functions", mimeo, Department of Economics, University of Colorado.

Varian, H. (1992). Microeconomic Analysis, third edition, W.W. Norton, New York.

Whalley, J. (1987). "Operationalizing Walras: Experience with Recent Applied General Equilibrium Tax Models." Symposium paper presented to 5th World Meetings of the Econometric Society, Advances in Econometrics, Volume 2, Truman Bewley (ed.), (Cambridge, Cambridge University Press). 